\title{
Identical Service for Consistently Located Nodes with Different Disciplines of Service in Conditions of Heavy Traffic
}

\author{
Andrey Pavlov V. \\ Higher Mathematics,Moscow Institute of Radio-technics,Electronics and Automatics,78,pr.Vernadskogo, 119454 ,Moscow,Russia \\ *Corresponding Author: login11@umail.ru
}

Copyright (C2013 Horizon Research Publishing All rights reserved.

\begin{abstract}
We consider $\mathrm{K}$ nodes in series in conditions of identical service : the time of service of one customer on one device of nodes is the same for all different nodes (a.s.) . In every node $\mathrm{N}$ devices are located, $N \geq 1$. Each node contains the infinite set of places for wait . The tonal number of customers, the time of wait in nodes $2, \ldots, \mathrm{K}$ remains limited for all moments of times in heavy traffic conditions on the first node, if $\rho>1, \rho=1, \rho \rightarrow 1$. The fact takes place, if the time of service of one customer by one device of node less than constant. Similar results are got in the general situation and for disciplines of service "without interruption".
\end{abstract}

Keywords Identical Service, Multi-channels Nodes in Series Service Disciplines without Interruption, Heavy Traffic Diffusion Approximation, High loading Waiting Times

\section{Introduction}

We consider $\mathrm{K}$ nodes in series in conditions of identical service : the time of service of one customer on one unit ( device) of nodes is the same for all different nodes (a.s.) . In every node $\mathrm{N}$ devices are located, $N \geq 1$.Each node contains infinite set of places for wait .

Input process for the $j$ th node is the output process for the $(j-1)$ th node, $j=2, \ldots K$.

The identical time of service of any customers in the different nodes by every device is main restriction of the article:

$$
\xi_{j}^{1}=\xi_{j}^{2}=\ldots=\xi_{j}^{K}, j=1,2, \ldots,
$$

where the random value $\xi_{j}^{i}$ is the time of service of the $j$ th customer on the $i$ th node by one device ( unit of service ) ; all the different devices on all nodes are identical and $\left\{\xi_{j}^{1}, j=1,2, \ldots\right\}$ are mutually independent random values with the distribution function $F(x)=\operatorname{Pr}\left(\xi_{j}^{1} \leq x\right)$ . Other words, $\xi_{j}^{1}$ is the time of service of customer arriving on the first node $j$ th on the account ( for the ordinary input process ). If a group of customers arrived on node in some moment of time, the customers are disposed in group in the random order - all results d't rely on order in the group. (The exact definitions are considered in the second section ).

The main result can be formulated as follows : in the condition of identical service the total quantity of customers on nodes with numbers $2, \ldots, \mathrm{K}$ ( not one ) remains limited for all moments of times $t \in[0, \infty)$, if even the classical loading $\rho$ for the first node is more than one $(\rho>1)$ as so , if $\rho=1, \rho \rightarrow 1$ ( the third and fourth sections and the (8) expression ).

A similar situation takes place in conditions of diffusion approximation (Billingsley ([1])) for the first node ( the theorem 4.2 in the 4 -th part).

New methods of given article ( the (8) estimation ) were formulated in the work of Pavlov ([2]) first.

Some optimum systems for the simple networks as in our article were explored in works of Iashkov ([3]) ( the "processor-sharing" discipline ore EPS ), of Pavlov ([4-5]) ("the short requests are served before long" ore SRPT,SPT disciplines),of Boxma ([6]) - ( the identical service). For one node the use of SRPT or SPT(SJT) disciplines minimizes a number of customers in the systems. The disciplines was investigated in the works of Pavlov ([5]) and Franken,Konog,Arndt,Schmidt ([7]). Different optimum systems were considered in Shrage works ( ([8]) for instance).

The use of SRPT-SJF(SPT) disciplines reduces in $y(\rho)$ times the number of customers in the $G I|G I| N \mid \infty$ system and in some nets ( the works of Pavlov $([4,5,9]$ ) ).( The definition of the $G I|G I| N \mid \infty$ system can be found in work of Klimov ([10]) ).

In the works the exact expression of $y(\rho)$ is resulted in conditions of diffusion approximation and, if $\rho \rightarrow 1-0$. The time of service (the definition is below ) $V_{1}^{\infty}<\infty$ (a.s.) for the $M|G I| 1 \mid \infty$ system with the SRPT discipline,if the load $\rho=1$ ( but $E V_{1}^{\infty}=\infty$ ) in work of Pavlov ([4]). The description of main works concerning the optimal disciplines of service ( from 1974 to 1985 yy.) can be found in the review article of Pechincin, Solovjev ([11]). Some models with the identical service are studied in the works of Boxma $([6])$ and Vinogradov $([12])$. 
In our article for $j>1$ we explore a net characteristics

$W_{j}^{t}$ - the total time of service of all the customers being on $j$-th node at t moment , $j=1, \ldots, K$, ( the virtual time of wait for one channel );

$\nu_{j}^{t}$ - the total number of the customers on $\mathrm{j}$-th node at t moment $, j=1, \ldots, K$;

$V_{j}^{t}$ - the full time of service on the $\mathrm{j}$-th node of the customer, which arrives on the $j$-th node at t moment $j=1, \ldots, K$ ( the time of wait plus the "length of customer").

We suppose $N=1$ in the first part of the work (the 3-th section ) and $N>1$ in the fourth sections.

In the fifth section the previous results are generalized for a general class of disciplines of service, but for one characteristic $W_{j}^{t}, j>1$. The class contains all disciplines, which satisfy to the requirement : any customer is served by the appointed device without interruption from start to finish. In different nodes ( in first node too ) can be different disciplines from the class - all results of previous sections hold good, but only for $W_{j}^{t}, j>1$.

All the results of sections 3,4 ( for the nodes with numbers $2, \ldots, K)$ take place in different situations of "heavy traffic" too : as for $\rho \rightarrow 1-0$ so for $\rho=1, \rho>1$ ( sections 3 and 4$)$, and, if $(\lambda(T)-\mu(T)) \sqrt{T} \rightarrow c,-\infty \leq c \leq+\infty$ ( the theorem 4.2), where, by definition, $\rho=\lambda(T) / \mu(T)$; $\lambda(T)$ - the intensity of the process of arriving of customers on the first node, $\mu(T)=N / E \xi_{1}^{1}$ - the intensity of service for the first node ( sections 3 and 4 ). The system and its characteristics depends on the parameter $T \rightarrow \infty$.

Only restriction concerning the arrival process of customers on the first node is the (2) condition. The condition is executed for all known random processes.

Of principle part at proof of all results is acted by (7) estimation. From the formula we obtain main results of our article :

$$
\begin{gathered}
\max _{j=2, \ldots K} \max _{t \leq T} \sup _{t \leq T}\left(W_{j}^{t}, V_{j}^{t}\right) \leq(2 N-1) \max _{j \leq A(T)} \xi_{j}^{1}, \\
\sup _{t \leq T, j \geq 2}\left(W_{j}^{t}, V_{j}^{t}\right) \leq \max _{j} \xi_{j}^{1}<C_{0}=\text { const. }
\end{gathered}
$$

if

$$
\max _{j} \xi_{j}^{1}<C_{0}
$$

(the theorems $3.1,4.1$ ), where $A_{T}(t)$ is the quantity of customers arriving on the first note during $[0, t]$. In lemma 3.1 the estimation of the given maximum is resulted.

\section{Main definitions .}

We consider the $K$ nodes, $K \geq 2$. The arrival process for the $j$-th node is equal to the output process on the $(j-1)=$ th node, $j=2, \ldots K$.

In the first node the customers are served in order of arrival,and as soon as the service is finished the customer arrives on the next node. In all nodes the customers are served in order of arrival. If a customers arrives on a node in group (for non-ordinary process ), the customers are disposed in the group in the random order - all results $d^{\prime} t$ rely on order in the group.
The $j$-th node consists of $\mathrm{N}$ service units (devices) with infinite set of waiting places, $j=1, \ldots K$.

We suppose $N=1$ in the sections 3 , and $N>1$ in the sections 4,5 .

By definition, $A_{T}(t)$ is a number of final customer among all customers arriving on first node during $[0, t]$. (If the customers arrives on a node in group ,the customers are disposed in the group in random order). For the ordinary process

$$
A_{T}(t)=\max k: t_{k} \leq t, A_{T}(t)=0, \text { if } t_{1}>t,
$$

where $t_{n}$ is the moment of arriving on the first node of n-th customer.

We suppose, that

$$
A_{T}(T) / \lambda(T) T \stackrel{p}{\longrightarrow} 1, T \rightarrow \infty,
$$

where $\lambda(T)$ is the non-random function, such that $0<c_{1}<\sup _{T} \lambda(T)<c_{2}<\infty, c_{1}, c_{2}=$ const. ; $A_{T}(T t)$ belongs to $D[0,1], 0 \leq t \leq 1$. The process $A_{T}(T t), t \in[0,1]$, is determined on the probabilistic space $\left(D[O, 1], U_{0}, P\right), U_{0}=B_{D[0,1]}$, (the works of Billingsley ([1]) and Borovkov ([13]) ).

Let $j$ be the number of the customer arriving on the first node $\mathrm{j}$-th on the account. By definition, $\xi_{j}^{i}$ is the time of service of the $j$ th customer on the $i$ th node by one device ( unit of service ) ; all the different devices on all nodes are identical and $\left\{\xi_{j}^{1}, j=1,2, \ldots\right\}$ are mutually independent random values with the distribution function $F(x)=\operatorname{Pr}\left(\xi_{j}^{1}(T) \leq x\right)$ for all $T$.

Further we will use for $\xi_{j}^{i}$ the term "length of customer" too.

We suppose

$$
\xi_{j}^{1}=\xi_{j}^{2}=\ldots=\xi_{j}^{K},
$$

for all $j=1,2, \ldots$ ( the condition of identical service ).

The process $A_{T}(t T), 0 \leq t \leq 1$, and the sequence $\left\{\xi_{j}^{1}(T), j=1,2, \ldots\right\}$ are mutually independent for all $T \in[0, \infty)$.

Further we will skip the parameter $T$ in the designations .

We suppose in the 4.2 theorem

$$
\sup _{T} E\left(\xi_{1}^{1}(T)\right)^{2+\varepsilon}<c, c, \varepsilon-\text { const., } c<\infty, \varepsilon>0 .
$$

$$
\mu(T)=N / E \xi_{1}^{1}(T), \rho(T)=\lambda(T) / \mu(T) .
$$

By definition,

$$
1-F_{T}(x)=\bar{F}(x)
$$

$x \in[0, \infty)$, and

$$
\Phi(\delta)=\inf x: 1-F_{T}(x) \leq \delta, 0<\delta<1 ; \bar{F}(\Phi(\delta))=\delta,
$$

for the continuous $F_{T}(x)$.

In all sections we will use following two conditions : if $\sup _{T} F_{T}(x)<1$ for all $x>0$, if $\sup _{T} E \xi_{1}^{1}(T)<\infty$, we use

$$
\sup _{T} \bar{F}_{T}(x)<\gamma_{1} / x^{1+\gamma}, x \rightarrow \infty, \gamma>0,
$$

$\gamma_{1}, \gamma=$ const

Other condition

$$
\lim _{x \rightarrow \infty} \sup _{T} \bar{F}_{T}(\beta x) / \bar{F}_{T}(x)=0,
$$

for all $\beta>1, \beta=$ const., $\beta<\infty$. 


\section{The identical service for single channel in each node and the discipline FIFO.}

In the section we study our characteristics for the FIFO discipline and for one device in each node , $N=1$. (FIFO - serving in order of arriving (the works of Klimov ([10]) and Borovkov ([14] ).

The main result in theorem 3.1 is formulated for all $N=1, \ldots, K$ ( the proof for $N>1$ resulted in section 4).

Let $\nu_{j}^{0}=0$, and $N=1$ for all $j=1, \ldots, K$.

Theorem 3.1

1 .

$$
\begin{gathered}
\max _{i=2, \ldots, K} \sup _{o \leq t \leq T} \max \left(W_{i}^{t}, V_{i}^{t}\right) \leq C(N) \Delta(T), \\
\Delta(t)=\max _{j \leq A_{T}(t)} \xi_{j}^{1}, C(N)=2 N-1 ;
\end{gathered}
$$

2.

$$
\begin{gathered}
E \chi\left(B_{1}(T)\right) \Delta(T) \leq c_{*} \Phi(1 / \lambda(T) T)(1+o(1)), \\
\lim _{t \rightarrow \infty} \operatorname{Pr}\left(B_{1}(T)\right)=1, B_{1}(T) \subset U,
\end{gathered}
$$

where $c_{*} \leq 2+1 / \gamma$, if the (4) condition takes place, and $c_{*}=1+o(1), T \rightarrow \infty$,if only the (5) condition takes place ;

3.

$E \nu_{j}^{T} \chi\left(B_{2}(T)\right) \leq \mu C(N)(j-1) c_{*} \Phi(1 / \lambda(T) T)(1+o(1))$, $j=2, \ldots, K$, if $\Phi(1 / \lambda(T) T) \rightarrow \infty$, and the (4) condition takes place, where $\lim _{T \rightarrow \infty} \operatorname{Pr}\left(B_{2}(T)\right)=1$, and $C(N)=$ 1 ,if $N=1(C(N)=2 N-1$, if $N>1$ as in the section 4).

Proof.

Let $A(j, t)$ be the number of the last customer arriving on the j-th node during $[0, t],\left(A(1, t)=A_{T}(t)=A(t)\right)$.

For $N=1$ we obtain from work of Borovkov $([14, \mathrm{p} .41])$

$$
\begin{gathered}
W_{j}^{t}=S_{j}(t)-t-\inf _{0 \leq u \leq t}\left(S_{j}(u)-u\right) \\
S_{j}(t)=\sum_{j=1}^{A(j, t)} \xi_{i}^{1}, j=1, \ldots, K
\end{gathered}
$$

At the $\mathrm{T}$ moment on the $(\mathrm{j}-1)$ the node there is a customer with number $n(t)$. By definition, $\theta_{j-1}^{t}$ is the part of the "length of customer" with number $n(t)$ is already served at $\mathrm{t}$ moment; $\theta_{j-1}^{t}=0$,if $W_{j}^{t}=0$ $, j-1=1, \ldots, K$.

We have

$$
S_{j}(t)=S_{j-1}(t)-\left(W_{j-1}^{t}+\theta_{j-1}^{t}\right), \theta_{j-1}^{t} \leq \xi_{n(t)}^{j-1},
$$

$j=2, \ldots, K$.

We obtain

$$
\begin{gathered}
W_{j}^{t}= \\
S_{j-1}(t)-t-\left[S_{j-1}(t)-t-\inf _{0 \leq u \leq t}\left(S_{j-1}(u)-u\right)\right]- \\
\theta_{j-1}^{t}-\inf _{0 \leq u \leq t}\left[S_{j-1}(u)-u-\right.
\end{gathered}
$$

$$
\begin{gathered}
\left.\left.\left(S_{j-1}(u)-u-\inf _{0 \leq v \leq u}\left(S_{j}(v)-v\right)\right)-\theta_{j-1}^{u}\right)\right]= \\
\inf _{0 \leq u \leq t}\left(S_{j}(u)-u\right)- \\
\left.\inf _{0 \leq u \leq t}\left[\inf _{0 \leq v \leq u}\left(S_{j-1}(v)-v\right)-\theta_{j-1}^{u}\right)\right]- \\
\theta_{j-1}^{t}=\inf _{0 \leq u \leq t}\left(S_{j-1}(u)-u\right)- \\
\inf _{0 \leq v \leq u_{j}(t)}\left(S_{j-1}(v)-v\right)+\theta_{j-1}^{u_{j}(t)}-\theta_{j-1}^{t} \leq \\
\theta_{j-1}^{u_{j}(t)}-\theta_{j-1}^{t} \leq \Delta(t),
\end{gathered}
$$

where $u_{j}(t)$ is the moment, when the minimum of

$$
\left.\inf _{0 \leq u \leq t}\left[\inf _{0 \leq v \leq u}\left(S_{j-1}(v)-v\right)-\theta_{j-1}^{u}\right)\right]-\theta_{j-1}^{t}=a
$$

is equal to $a$ ( minimum by $t$ ).

From $u_{j}(t) \leq t, S_{j-1}(0)=0$ and $\theta_{j-1}^{t} \geq 0$ we get

$$
W_{j}^{t} \leq \theta_{j-1}^{u_{j}(t)}-\theta_{j-1}^{t} \leq \xi_{n\left(u_{j}(t)\right)}^{j-1} \leq \Delta(t) .
$$

We obtain ( from the (2) conditon )

$\chi\left(B_{1}(T)\right) A_{T}(T) \leq \lambda(T) T(1+o(1))=R(T), T \rightarrow \infty$,

$\lim _{T \rightarrow \infty} \operatorname{Pr}\left(B_{1}(T)\right)=1$,

The lemma 3.1 may us need.

Lemma 3.1.

1 .

$$
E \max _{1 \leq i \leq n} \xi_{i}^{1} \leq(2+1 / \gamma) \Phi(1 / n)(1+o(1))
$$

if the (4) condition takes place $(\bar{F}(y(n))=1 / n, y(n)=$ $\Phi(1 / n) \rightarrow \infty, n \rightarrow \infty$, for continuous $\bar{F}(x): \bar{F}(x)<1$ for all $x \in[0, \infty))$;

2 .

$$
E\left(\max _{1 \leq i \leq n} \xi_{i}^{1}\right)^{q}=(\Phi(1 / n))^{q}(1+o(1))
$$

$n \rightarrow \infty, q \geq 1$,if only the (5) condition takes place.

Proof.

By definition, a constant $\alpha=1$, if the (4) condition takes place, and the constant $\alpha \geq 1$, if only the (5) condition takes place. ( The works of Pavlov ([5]) and Harrison ([15]) ).

We use the equality

$$
\begin{gathered}
\bar{F}(y)=\bar{F}(y+0) \leq 1 / n \leq \bar{F}(y-0), \Phi(1 / n)=y(n), \\
\bar{F}(\Phi(\delta))=\delta, \delta \in[0, \infty),
\end{gathered}
$$

to define a function $y(n) ; n \leq 1 / \bar{F}(y)$, ( for the continuous $F(t), F(y(n))=1 / n, \Phi(1 / n)=y(n))$.

We get

$$
\begin{gathered}
m=\max _{1 \leq i \leq n} \xi_{i}^{1} \leq \alpha y \operatorname{Pr}\left(\max _{1 \leq i \leq n} \xi_{i}^{j} \leq \alpha y\right)+ \\
+\int_{\alpha y+0}^{\infty} x d\left(F^{n}(x)\right) \\
\leq \alpha y\left(1+\int_{\alpha y+0}^{\infty} t d F(t) / \alpha y \bar{F}(y)\right)= \\
=\alpha y\left(1+(\bar{F}(\alpha y) / \bar{F}(y))\left[1+\int_{\alpha y+0}^{\infty} \bar{F}(t) d t / \alpha y \bar{F}(\alpha y)\right]\right) .
\end{gathered}
$$


By analogy with the Cauchy theorem we have (from the condition (4))

$$
\begin{gathered}
x \bar{F}(x) / \int_{x}^{\infty} \bar{F}(t) d t= \\
-d\left(\ln \int_{x}^{\infty} \bar{F}(t) d t\right) / d x / d(\ln x) / d x= \\
=-\ln \left(\int_{\theta}^{\infty} \bar{F}(t) d t\right) / \ln \theta=\log _{\theta}\left(\int_{\theta}^{\infty} \bar{F}(t) d t\right)^{-1} \geq \\
\geq \log _{\theta}\left(\int_{\theta}^{\infty} \gamma / t^{1+\gamma} d t\right)^{-1}= \\
=\log _{\theta} \theta^{\gamma}=\gamma, \\
x=\alpha y, \theta>\alpha y, \int_{x}^{\infty} \bar{F}(t) d t \rightarrow 0, \theta \geq x \rightarrow \infty
\end{gathered}
$$

as $n \rightarrow \infty$.

We obtain

$$
\begin{gathered}
m \leq \alpha y(1+(\bar{F}(\alpha y) / \bar{F}(y))(1+1 / \gamma)) \leq \\
2 \alpha y(1+1 / \gamma)
\end{gathered}
$$

$n \rightarrow \infty$. The (9) expression is proved for the constants $\alpha=1$, and the first part of the lemma is proved.

Now, we use the $(5)$ condition. We can use the (9) equality for a constant $\alpha>1$. We obtain $m \leq \alpha y(1+$ $o(1))$ or

$$
m \leq y(n)(1+o(1), \text { as } n \rightarrow \infty,
$$

(From the (5) condition we have the (4) condition with the constant $\gamma \geq 0$.)

To obtain $m \geq \alpha_{1} y(1+o(1))$ with $\alpha_{1}<1$ we use

$$
m \geq \operatorname{Pr}\left(\max _{i \leq n} \xi_{i}^{1}>\alpha_{1} y\right) \alpha_{1} y,
$$

and

$$
\begin{aligned}
& \operatorname{Pr}\left(\max _{i \leq n} \xi_{i}^{1}>\alpha_{1} y\right)=1-\exp \left(n \ln \left(1-\bar{F}\left(\alpha_{1} y\right)\right)=\right. \\
& =1-\exp \left[-(1+o(1)) \bar{F}\left(\alpha_{1} y\right) / \bar{F}(y)\right]=1+o(1),
\end{aligned}
$$

$n \rightarrow \infty, y(n) \rightarrow \infty, \alpha_{1}=$ const., $\alpha_{1}<1$, ( in last equality we use the (5) condition ).

We obtain $m \geq y(n)(1+o(1)), n \rightarrow \infty$. For $q=1$ the second part of lemma is proved.

If $q>1$, we use the equalities

$$
\begin{gathered}
\left(\max \xi_{i}^{1}\right)^{q}=\max \left(\xi_{i}^{1}\right)^{q}, \\
\bar{G}(x)=\operatorname{Pr}\left(\left(\xi_{i}^{1}\right)^{q}>x\right)=\bar{F}\left(x^{1 / q}\right) .
\end{gathered}
$$

For $\bar{G}(x)$ the $(5)$ condition is the (5) condition for $\bar{F}(x)$, and we have

$$
E \max _{i \leq n}\left(\xi_{i}^{1}\right)^{q} \sim y^{q}, n \rightarrow \infty, q>1 .
$$

The proof of the lemma 3.2 is complete. ( We used the methods of Pavlov $([4,5,16])$.

From lemma 3.1 we get

$$
\begin{gathered}
E \chi\left(B_{1}(T)\right) \Delta(T) \leq E \max _{i \leq R(T)} \xi_{i}^{1} \leq \\
\left.c_{*}(1+o(1)) \Phi(1 / \lambda(T)) T\right)=o\left(T^{1 / 2}\right),
\end{gathered}
$$

We obtain the theorem 3.1 for $W_{j}^{T t}, N=1$ ( we use the (8) equality ).

Now we consider $V_{j}^{t}$, if $N=1$.

From the definition of $\pi(j, t)$

$$
V_{j}^{t}=W_{j}^{\pi(j, t)},\left(W_{j}^{t}=W_{j}^{t+0}\right), t \geq 0,
$$

where $\pi(j, t)$ is the moment of arrival of customer with the number $A_{T}(t)$ on the $j$-th node.

We can use the (7) and (8) equalities with $\pi(j, t)$ instead of $t$. We have

$$
V_{j}^{t} \leq \sup _{u \leq \pi(j, t)} \theta_{j-1}^{u} \leq \sup _{j \leq A(t)} \xi_{j}^{1}=\Delta(t) \leq \Delta(T), t \leq T,
$$

and

$$
\sup _{0 \leq t \leq T} V_{j}^{t} \leq \Delta(T)
$$

By analogy with $W_{j}^{t}$ we obtain the theorem 3.1 for $V_{j}^{t}$, (from the (10) equality and the 3.1 lemma ).

Now we consider $\nu_{j}^{t},(N=1)$.

Let $A(j, t)$ be the number of the last customer arriving on the $\mathrm{j}$-th node during $[0, t],\left(A(1, t)=A_{T}(t)=A(t)\right)$.

The customer on the $\mathrm{j}$-th node at $\mathrm{t}$ moment has the number $r(j, t)$, and $\tau(t, j)$ is the moment of arrival of the customer with such number on the $j$-th node.

We can write

$$
\begin{gathered}
\chi\left(\nu_{j}^{t}>0\right) \nu_{j}^{t}= \\
\chi\left(\nu_{j}^{t}>0\right)\left[A_{T}(j, t)-A_{T}\left(j, t-W_{j}^{\tau(t, j)+}+\xi_{r(j, t)}^{1}-\theta_{j}^{t}\right)\right] \leq \\
\leq A(j, t)-A\left(j, t-W_{j}^{\tau(t, j+)}\right),
\end{gathered}
$$

With help the (8) equality

$$
\sup _{0 \leq v \leq t} W_{j}^{\tau(v, j)} \leq \sup _{0 \leq s \leq \tau(v, j)} \theta_{j-1}^{u_{j}(s)} \leq \Delta(t) .
$$

And from

$$
A(j, t)=A(j-1, t)-\nu_{j-1}^{t}
$$

we get

$$
\begin{gathered}
A(j, t)-A\left(j, t-W_{j}^{\tau(t, j)}\right) \leq A(j, t)-A(j, t-\Delta(t)) \leq \\
A(j-1, t)-\nu_{j-1}^{t}-\left[A(j-1, t-\Delta(t))-\nu_{j-1}^{t-\Delta(t)}\right],
\end{gathered}
$$

where

$$
\begin{gathered}
\nu_{j-1}^{t-\Delta(t)} \leq A(j-1, t-\Delta(t))- \\
-A\left(j-1, t-\Delta(t)-W_{j-1}^{\tau(t-\Delta(t), j-1)}\right),
\end{gathered}
$$

and

$$
W_{j-1}^{\tau(t-\Delta(t), j-1)} \leq \Delta(t)
$$

or

$$
\begin{gathered}
\chi\left(\nu_{j}^{t}>0\right) \nu_{j}^{t} \leq A(j-1, t)-A(j-1, t-2 \Delta(t)) \leq \ldots \leq \\
A(2, t)-A(2, t-(j-1) \Delta(t)) .
\end{gathered}
$$

We obtain ( from the (10) equality )

$$
\begin{gathered}
\chi\left(B_{1}(T)\right) \Delta(T) \leq \\
c_{*}(1+o(1)) \Phi(1 / \lambda(T) T), \lim _{T \rightarrow \infty} \operatorname{Pr}\left(B_{1}(T)\right)=1 .
\end{gathered}
$$


By definition,

$\varphi(T)=(j-1) c_{*}(1+o(1)) \Phi(1 / \lambda(T) T), w=T-\varphi(T)$,

and

$$
\begin{gathered}
\chi\left(B_{1}(T)\right)[A(2, T)-A(2, T-(j-1) \Delta(T))] \leq \\
A(2, T)-A(2, w) .
\end{gathered}
$$

( We repeat the methods of work of Pavlov ([5])).

We will use the designations

$$
\begin{gathered}
H(\phi(T))= \\
\min n:\left(\xi_{A(2, w)+1}^{1}-\theta_{1}^{w}\right)+\sum_{i=A(2, w)+2}^{A(2, w)+n} \xi_{i}^{1} \leq \\
T-w=\varphi(T),
\end{gathered}
$$

if $n \geq 0$,and

$$
H(\varphi(T))=0
$$

if

$$
\xi_{A(2, w)+1}^{1}-\theta_{1}^{w}>T-w \bigcup A(2, T)-A(2, w)=0 .
$$

The random values $A(2, w)$ and $\left\{\xi_{i}^{1}, i \geq A(2, w)+2\right\}$ are independent :

$$
A(2, T)-A(2, w) \leq H(\varphi(T))+1,
$$

where $H(t)$ is the process of renewal generated by $\left\{\xi_{j}^{1}, j \geq A(2, w)+2\right\}$.

With help the $(2)$ condition we get

$$
\begin{gathered}
E \chi\left(B_{3}(T)\right) H(\varphi(T)) \leq \\
E \chi\left(B_{3}(T)\right)[(H(\varphi(T))-\mu(T) \varphi(T)) \vee 0]+ \\
+\mu(T) \varphi(T)=\mu(T) \varphi(T)(1+o(1)), \\
\mu(T)=1 / E \xi_{1}^{1}, a \vee b=\max (a, b), \varphi(T) \rightarrow \infty . \text { We use } \\
\lim _{T \rightarrow \infty} \operatorname{Pr}\left(B_{3}(T)\right)=1, \\
B_{3}(T)= \\
\{w:|[H(\varphi(T))-\mu(T) \varphi(T)] / \sqrt{\varphi(T)}|<\ln \varphi(T)\} .
\end{gathered}
$$

Now (from (13),(14) and (15) ), we have

$$
\begin{gathered}
E\left(\chi\left(B_{1} \cap B_{3}\right) \nu_{j}^{T} \leq \mu(T) \varphi(T)(1+o(1)),\right. \\
B_{2}=B_{1} \cap B_{3}, \operatorname{Pr}\left(B_{3}\right) \rightarrow 1, T \rightarrow \infty .
\end{gathered}
$$

The proof of the theorem 3.1 is complete.

\section{The identical service for $N$ channels and FIFO discipline in each node.}

We will prove a theorem in supposition, that every node consists of $\mathrm{N}$ devices, $N>1$.

Theorem 4.1.

All results of the theorem 3.1 take place with $C(N)=$ $2 N-1, N>1$.

Proof.

We introduce a new $Q_{j}(N)$ system on the $j$-th node with help $\mathrm{S} 1$ and $\mathrm{S} 2, j=1, \ldots, K$.

The $\mathrm{S} 1$ conditions.

For the $Q_{j}(N)$ system the arrival process is equal $A(j, t), j=1,2, \ldots, K$, as in main $\mathrm{j}$-th node ( we determined $A(j, t)$ in the 3.1 theorem too).

The "length of customer" of all customers in the $Q_{j}(N)$ system equals to the lengths for the main system .

The $\mathrm{S} 2$ conditions .

The speed of service in the $Q_{j}(N)$ system is $\mathrm{N}$ (the $j$ th node of the $Q_{j}(N)$ system contains one device and the speed of work on the device is $N), j \geq 1$.

Let $w_{j}^{t}$ is a sum of remaining "lengths of all customer" on the $\mathrm{j}$-th node at the $\mathrm{t}$ moment ( some of remaining lengths are equal to the complete lengths ).

By definition, for main system (not for $Q_{j}(N)$ system) $W_{* j}^{t}$ is a total sum of full "lengths of customers" on the $j$ th node at the t moment, $j=1, \ldots, K$. $\left(W_{* j}^{t}=\right.$ $W_{j}^{t}+\theta_{j}^{t}$,if $N=1$ ).

We can use the 2.2 lemma from work of Pavlov ([5]), and with help of additional part 6 we obtain

$$
\begin{gathered}
0 \leq W_{j}^{t}-w_{j}^{t} \leq(N-1) \Delta_{j}(t), \\
0 \leq W_{* j}^{t}-w_{j}^{t} \leq N \Delta_{j}^{t}, \\
\Delta_{j}(t)=\sup _{i \leq A(j, t)} \xi_{i}^{j}
\end{gathered}
$$

for all $t>0, j \geq 1$.

The speed of work is $N$ ( in the $Q_{j}(N)$ system), and we can use [14,p.41],

$$
w_{j}^{t}=S_{j}(t)-N t-\inf _{0 \leq u \leq t}\left(S_{j}(u)-N u\right)
$$

$j=1, \ldots, K$. (The definition of $S_{j}(u)$ was in the theorem $3.1)$.

We get (from the (17) and (6) equalities )

$$
\begin{gathered}
W_{j}^{t}=w_{j}^{t}+r_{j}(t), 0 \leq r_{j}(t) \leq(N-1) \Delta(t), \\
W_{* j}^{t}=w_{j}^{t}+r_{j}^{*}(t), 0 \leq r_{j}^{*}(t) \leq N \Delta(t), j \geq 1 ; \\
S_{j}(t)=S_{j-1}(t)-W_{* j-1}^{t}=S_{j-1}(t)-w_{j-1}^{t}-r_{j-1}^{*}(t), j \geq 2 .
\end{gathered}
$$

We use the (17),(18) equalities and

$\inf _{0 \leq u \leq t}\left(S_{j-1}(u)-N u\right)-\inf _{0 \leq u \leq t} \inf _{0 \leq v \leq u}\left(S_{j-1}(v)-N v\right)=0$, to obtain

$W_{j}^{t}=w_{j}^{t}+r_{j}(t)=S_{j-1}(t)-\left(w_{j-1}(t)+r_{j-1}^{*}(t)\right)-N t-$ $-\inf _{0 \leq u \leq t}\left[S_{j-1}(u)-\left(w_{j-1}^{u}+r_{j-1}^{*}(u)\right)-N u\right]+r_{j}(t) \leq$ 


$$
\begin{gathered}
\leq S_{j-1}(t)-w_{j-1}^{t}+\sup _{0 \leq u \leq t} r_{j-1}^{*}(u)-N t- \\
\inf _{0 \leq u \leq t}\left(S_{j-1}(u)-w_{j-1}^{u}-N u\right)+r_{j}(t)= \\
\inf _{0 \leq u \leq t}\left(S_{j-1}(u)-N u\right)-\inf _{0 \leq u \leq t} \inf _{0 \leq v \leq u}\left(S_{j-1}(v)-N v\right)+ \\
r_{j}(t)+\sup _{0 \leq u \leq t} r_{j-1}^{*}(u) \leq(2 N-1) \Delta(t)
\end{gathered}
$$

for all $t \leq T, j \geq 2$.

By definition, $v(j, t)$ is the moment of arriving of the customer with number $A(t)$ in the $j$ th node. We will replace t on $v(j, t)$ in (19) equality, and

$$
V_{j}^{t} \leq W_{j}^{v(j, t)}, j \geq 2
$$

(where $V_{j}^{t}=W_{j}^{v(j, t)}$,if $A(j, t)$ is the ordinary process). We obtain

$$
V_{j}^{t} \leq \sup _{0 \leq u \leq v(j, t)} r_{j-1}^{*}(u)+r_{j}(v(j, t)), j \geq 2 .
$$

We can use the 2.2 lemma from work of Pavlov ([5]), and with help of additional part 6 we get

$$
r_{j-1}^{*}(u) \leq N\left(\sup _{1 \leq i \leq A(j-1, r(j, t))} \xi_{i}^{1}\right) \leq N \Delta(t),
$$

$u \leq r(j, t)$

$r_{j}(r(j, t)) \leq(N-1)\left(\sup _{1 \leq i \leq A(j, r(j, t))} \xi_{i}^{1}\right) \leq(N-1) \Delta(t)$.

If the customer with number $A(t)$ arrives in $j$ th node $(j>1)$ later than a customer with a number $A(t)+m$, we use

$$
\xi_{A(t)+m}^{1} \leq \xi_{A(t)}^{1}, m \in 1,2, \ldots
$$

We proved

$$
V_{j}^{t} \leq(2 N-1) \Delta(t) \leq(2 N-1) \Delta(T) .
$$

We will consider $\nu_{j}^{T}$ for $N>1$.

We will define the $Z_{j}(t)$ set . The $Z_{j}(t)$ set consists of the customer accepted to service at $t$ moment ( not more than $N$ ).

By definition, $\tau(N, j, t)$ is a moment of arrival of last from customers from the $Z_{j}(t)$ set ( on the j-th node).

As in the (12) equality

$$
\chi\left(\nu_{j}^{t}>0\right) \nu_{j}^{t} \leq A(j, t)-A\left(j, t-W_{j}^{\tau(N, j, t+)}\right),
$$

where $W_{t}^{j} \leq(2 N-1) \Delta(t), t \leq T, j \geq 2$.

By analogy with the (12),(13) equalities from the (19) equality we obtain

$$
\nu_{j}^{t} \leq A(2, t)-A(2, t-(2 N-1)(j-1) \Delta(t)), j \geq 2 .
$$

By definition,

$$
\psi(T)=(2 N-1)(j-1) c_{*}(1+o(1)) \Phi(1 / \lambda(T) T) .
$$

We obtain by an analogy with the (15),(16),(14) equalities

$$
\left.E \chi\left(B_{2}(T)\right) \nu_{j}^{T} \leq E \chi\left(B_{2}(T)\right) \sum_{i=1}^{N}\left[H_{i}(\psi(T))+1\right)\right]=
$$

$$
\begin{gathered}
E \chi\left(B_{2}(T)\right)\left(\sum _ { i = 1 } ^ { N } \left[H_{i}(\psi(T))+1-\right.\right. \\
\mu(T) \psi(t) / N]+\mu(T) \psi(T))=\mu(T) \psi(T)(1+o(1)), \\
\lim _{T \rightarrow \infty} \operatorname{Pr}\left(B_{2}(T)\right)=1, \mu(T)=N / E \xi_{1}^{1},
\end{gathered}
$$

if $\psi(T) \rightarrow \infty, T \rightarrow \infty$, where $H_{i}(t), i=1, \ldots, N$ are the processes of renewal (independent). The $H_{i}(t)$ process is generated by a sequence distributed as $\left\{\xi_{j}^{1}\right\}$.

The theorem 4.1 is proved.

We use $\stackrel{D(K)}{\longrightarrow}$ for the definition of weak convergence in the functional space $D[0,1]^{K}$ ( Billingsley ([1]) ).

We use the definitions in the theorem $4.2: Z_{i}^{t}(1)=$ $\nu_{i}^{t}, Z_{i}^{t}(2)=W_{i}^{t}, Z_{i}^{t}(3)=V_{i}^{t}, i=1,2, \ldots, K$.

Theorem 4.2 .

$$
T^{-1 / 2}\left(Z_{1}^{T t}(J), \ldots, Z_{K}^{T t}(J)\right) \stackrel{D(K)}{\longrightarrow}\left(f_{J}(t), 0, \ldots, 0\right),
$$

$J=1,2,3$, if

1.

$$
\left.E \xi_{1}^{1} \rightarrow g>0, E\left(\xi_{1}^{1}\right)^{2}-\left(E(\xi)_{1}^{1}\right)\right)^{2} \rightarrow \sigma^{2}>0,
$$

$T \rightarrow \infty$, where $g, \sigma=$ const.

2 .

$$
\left(A_{T}(t T)-\lambda(T) T t\right) / T^{1 / 2} \stackrel{D(K)}{\longrightarrow} A^{0}(t) \subset D[0,1]
$$

if

$(\lambda(T)-\mu(T)) T^{1 / 2} \longrightarrow c_{0}, T \rightarrow \infty, c_{0}=$ const.,$-\infty<c_{0}<\infty$,

$$
E\left(\xi_{1}^{1}\right)^{2+\varepsilon}<\text { const. }<\infty, \varepsilon>0 .
$$

( The $f_{J}(t)$ process is well known for the most input processes ( on the first node) ).( Borovkov ([13,14]) ).

Proof.

From the (3) condition we get

$\sup _{T} \bar{F}_{T}(x) \leq c / x^{1+(1+\varepsilon)}, \gamma=1+\varepsilon>0, c=$ const.,$x \rightarrow \infty$.

By definition,

$$
B_{3}(T)=\{w: A(T)<\sqrt{T} \ln T+\lambda(T) T=\lambda(T) T(1+o(1))\},
$$

(we use the (2) condition).And from the 3.1 lemma we obtain

$$
\begin{gathered}
E \chi\left(B_{1}(T)\right) \Delta(T) \leq c_{*}(1+o(1)) \Phi(1 / \lambda(T) T)= \\
o\left(T^{1 / 2}\right), T \rightarrow \infty, \\
\lim _{T \rightarrow \infty} \operatorname{Pr}\left(B_{1}(T)\right)=1 .
\end{gathered}
$$

For the $Z_{2}^{T t}, Z_{3}^{T t}$ characteristics we have:

$\chi\left(B_{3}(T)\right) \sup _{t<1} Z_{j}^{T t}(J) / T^{1 / 2} \leq \chi\left(B_{3}(T)\right) \Delta(T) / T^{1 / 2} \stackrel{p}{\longrightarrow} 0$,

$j=2, \ldots, K$.

The theorem is proved for $Z_{J}^{T t}, J=2,3$. 
We will consider $\nu_{j}^{t}=Z_{1}^{t}$.

If $\psi_{0}=(2 N-1)(j-1) \Delta(T)$ in $(20)$, we get

$$
\begin{gathered}
\sup _{t \leq T} \nu_{j}^{t} \leq \sup _{t \leq T}\left|A(2, t)-A\left(2, t-\psi_{0}\right)\right|= \\
=\sup _{t \leq T}\left|A(1, t)-A\left(1, t-\psi_{0}\right)+\nu_{1}^{t-\psi_{0}}-\nu_{1}^{t}\right| \leq \\
\leq \sup _{|t-s|<\psi_{0}}\left(|A(t)-A(s)|+\left|\nu_{1}^{t}-\nu_{1}^{s}\right|\right), t, s \in[0, T],
\end{gathered}
$$

where $E \chi\left(B_{3}\right) \psi_{0}=o\left(T^{1 / 2}\right), T \rightarrow \infty,($ as in $(21))$.

$A_{T}^{*}(t)=A_{T}(t)-\lambda(T) t$, and we have

$$
\begin{gathered}
\chi\left(B_{1}\right) \sup _{t \leq T} \nu_{j}^{t} / T^{1 / 2} \leq \\
\sup _{|t-s|<o\left(T^{1 / 2}\right)}\left[\left(\left|A^{*}(t)-A^{*}(s)\right|+\lambda(T)(s-t)\right) / T^{1 / 2}+\right. \\
\left.\left(\nu_{1}^{t}-\nu_{1}^{s} \mid\right) / T^{1 / 2}\right]= \\
\delta_{j}(T),
\end{gathered}
$$

$T \rightarrow \infty, t, s \leq T, j \geq 0$.

It is well known ( Harrison ([15]), Iglehart and Whitt $([17]))$

$$
\nu_{1}^{T t} / T^{1 / 2} \stackrel{D(1)}{\longrightarrow} f_{1}(t) \subset D[0,1], T \rightarrow \infty .
$$

We use

$$
A_{T}^{*}(T t) \stackrel{D(1)}{\longrightarrow} A^{0}(t), T \rightarrow \infty .
$$

From the condition of the weak convergence (Billingsley $([1]))$, as in the work of Iglehart and Whitt ([17]), we obtain

$$
\delta_{j}(T) \stackrel{p}{\longrightarrow} 0, t \rightarrow \infty, j=2, \ldots, K .
$$

The 4.2 theorem is proved.

\section{Identical service for all disci- plines "without interruption of service".}

In the section we consider all the disciplines of service " without interruption of service.": any customer is served by the appointed device without interruption from start to finish (Pechincin, Solovjev ([11]),Schrage ([8]) ).

By definition, $d \in G$, if for the $d$ service discipline the conditions D1 and D2 take place.

\section{Condition D1.}

Speed of service is equal to unit (one) for every device, if the device works.

\section{Condition D2.}

After arriving on device any customer is served without interruption from start to finish .

Theorem 5.1.

All results of the theorems 4.1 and 4.2 take place for the characteristics $W_{j}^{t}, \mathrm{j}=2, \ldots, \mathrm{K}$, if $d_{j}$ discipline handles by service in the $\mathrm{j}$-th node, and $d_{j} \in G, j=1, \ldots, K$.( If $N=1$ or $N>1$ ).

( $W_{j}^{t}$ is the total sum of remaining lengths of service (remaining "lengths of customers") for all customer on the $j t h$ node at the $\mathrm{t}$ moment ).
Proof.

By definition, $W_{j}^{t}=W_{j}^{t}\left(d_{j}\right)$ is the total sum of remaining lengths of service for all customer on the $j t h$ node at the $\mathrm{t}$ moment, $d_{0}$ is the FCFO (FIFO) discipline ( serving in order of arrival).

For $N=1$ we can write

$S_{j}(t)=\sum_{k=1}^{A(j-1, t)} \xi_{i_{k}}^{1}-W_{j-1}^{t}\left(d_{j-1}\right)-\theta_{j-1}^{t}, j=2, \ldots, K$,

where $i_{k} \neq i_{r}$, if $k \neq r$;

$$
W_{j-1}^{t}\left(d_{0}\right)=W_{j-1}^{t}\left(d_{j-1}\right), t>0, j=2, \ldots, K,
$$

if for the (j-1)-th node the arrival process is equal to $A(j-1, t), A(1, t)=A_{T}(t), k=1, \ldots, A(j-1, t)$.

The definition of the $\theta_{j}^{t}$ is in the 3 section (the theorem 3.1 - the (6),(7) conditions ).

We get a theorem 5.1 after the repetition the (7),(8),(10) equalities for $N=1$.

For $N>1$ the (17) estimation remains correct ( the woks of Pavlov $([5,18])$ and of Makarichev ([19]) ) for the disciplines of service $d_{j} \in G, j \geq 1$. We defined all values of the (17) equalities in the 3,4 sections and in the additional section 6 .

We obtain

$$
\begin{gathered}
W_{j}^{t}\left(d_{j}\right)=w_{j}^{t}+r_{j}(t), r_{j}(t) \leq(N-1) \sup _{i \leq A(j, t)} \xi_{i}^{j}, \\
W_{* j}^{t}\left(d_{j}\right)=w_{j}^{t}+r_{j}^{*}, r_{j}^{*}(t) \leq N \sup _{i \leq A(j, t)} \xi_{i}^{j}, j=1, \ldots, K .
\end{gathered}
$$

We get

$$
W_{j}^{t}\left(d_{j}\right) \leq(2 N-1) \Delta(T), t \leq T, j=2, \ldots, K,
$$

as in the 3,4 sections (from the (18),(19),(20) equalities ) .

Now,we can use the (21) equality and the 3.1 lemma, and the theorem 5.1 ( about $Z_{i}^{t}(2)$ as in the 4.2 theorem) is proved as in theorem 4.2.

\section{Appendix}

We consider a special estimation of $W_{j}^{t}$.

By definition, $W_{* j}^{t}$ is the total "length of customers" in j-th node at the $\mathrm{t}$ moment. If $N=1$

$$
W_{* j}^{t}=W_{j}^{t}+\theta_{j}^{t}, j=1, \ldots, K .
$$

Lemma 6.1.

$$
0 \leq \triangle W_{j}^{t}=W_{* j}(t)-w_{j}(t) \leq N \sup _{i \leq A(j, t)} \xi_{i}^{j}
$$

if $N>1, j=1, \ldots, K$,

(For the $Q_{j}(N)$ system $w_{j}(t)$ defined in the theorem 4.1.)

Proof. We will skip an index $j$.

By definition, $0=t_{1}<s_{1}<t_{2}<s_{2}<\ldots<t_{k}<$ $s_{k}<\ldots$, are such points, that $\nu_{j}^{t} \leq N-1$, if $t \in\left[t_{k}, s_{k}\right)$, $\nu_{j}^{t} \geq N$, if $t \in\left[s_{k}, t_{k+1}\right), k=1,2, \ldots$

We get

$$
\triangle W^{t} \leq(N-1) \sup _{i \leq A(j, t)} \xi_{i}^{j}
$$


if $t \in\left[t_{k}, s_{k}\right), k=1,2, \ldots$.

We will use $s(i)$, where $s(i)$ is a moment of ending of service of a customer in $\mathrm{j}$-th node,$k \geq 1$.

(The customer leaves from the $\mathrm{j}$-th node at $s(i)$ ). $\{s(i), i=1, \ldots, \rho\}$ are all the such moments between $s_{k}$ and $t_{k+1}, s_{k}=s(0) \leq s(1) \leq \ldots \leq s(\rho)=t_{k+1}$.

We have

$$
\begin{gathered}
W_{* j}^{s(i)}=W_{* j}^{s(i)+0}= \\
W_{j}^{s(0)-0}+S_{j}(s(i))-S(s(0)-0)-\sum_{m=1}^{i} \xi_{j_{m}} .
\end{gathered}
$$

The "length of customer" is more than remaining length, therefore we have

$$
\begin{gathered}
w_{j}^{s(i)}=w_{j}^{s(i)+0}= \\
w_{j}^{s(0)-0}+S_{j}(s(i))-S_{j}(s(0)-0)-\sum_{m=1}^{i} \xi_{j_{m}}^{*},
\end{gathered}
$$

where

$$
0 \leq \sum_{m=1}^{i} \xi_{j_{m}}^{*} \leq \sum_{m=1}^{i} \xi_{j_{m}}
$$

and

$$
\begin{gathered}
\triangle W^{s(i)+0}= \\
\triangle W^{s(i)-0}-w_{j}^{s(0)-0} \leq(N-1) \sup _{m \leq A(j, s(0))} \xi_{m}^{j}
\end{gathered}
$$

for all $i=1, \ldots, \rho$.

For $t \in(s(i-1), s(i)), i=1, \ldots, \rho$ we have

$$
\begin{gathered}
\triangle W^{t} \leq(N-1) \sup _{m \leq A(j, s(0))} \xi_{m}^{j}+\xi_{j_{i}} \leq \\
\leq N \sup _{m \leq A(j, t)} \xi_{m}^{j}
\end{gathered}
$$

for all $t \in(s(i-1), s(i))$, and,therefore,for all $t \in$ $\left[s_{k}, t_{k+1}\right)$.

\section{REFERENCES}

[1] P. Billingsley The convergence of probability measures.(in russian). Russia, Moscow: Nauka.1977, 352 pp.

[2] Pavlov A.V. Diffusion approximations and variation of conditions of ergodicity for identical service. Russia,Moscow:Adv.Math.Scien. 1997, v.52 , 3(315), p. 171 -172 .

[3] Iashkov S.F The analysis of a service system in IBM.(in russian) Russia, Moscow:Ed.Radio and Communic.1989, 216 c.

[4] Pavlov A.V. Some systems of service in heavy traffic. (In russian).Russia, Moscow : In Proc. of Seminar. "Stability Problems for Stochastic Models".1984, VNIISI , p. 87-92.
[5] Pavlov A.V The asymptotically optimal disciplines of service in the conditions of heavy traffic . Russia, Moscow: Theory Probab. and Appl. 1990.35,4, p. 713727.

[6] Boxma I.J. On a tandem service model with identical service time at both counters. UK. Adv.Appl.Probal.1979,11 ,p. 616-659.

[7] P.Franken,D.Konog,U.Arndt,V.Schmidt. The service systems and Point Processes.(in russian) Russia, Kiev.:Naukova Dumka.1981, 284 pp.

[8] Schrage L.E. The $M|G| 1$ systems with feedback to lower priority service. USA, Jour.Management science,1967, $13,7,466-474$.

[9] Pavlov A.V. The asymptotically optimal disciplines of service for some multi-channel networks.(In russian) Russia, Moscow: Theory Probab.and Appl.1991.36, 1, $170-175$.

[10] Klimov.G.P. Stochastic service systems. (In russian). Russia, Moscow: Nauka. 1966, 243 pp.

[11] Pechincin A.V., Solovjev A.D., An analysis and an optimization of the one-channel service system for a different disciplines .Russia, Moscow :In Proc. 3rd Intern.Sem.on Tel-traffic Theory, "Fundamentals of teltraffic theory". 1984.VINITI, p. 342-351.

[12] Vinogradov O.P. Two-phase system with identical service. Russia,Moscow:Nauka. Jour. Matem.zametki. 1983, 33,1, p. $141-146$

[13] Borovkov A.A. The asymptotic methods in a theory of service.(In russian).Russia,Moscow:Nauka.1980, 384 pp.

[14] Borovkov A.A. The stochastic process in a theory of service. (In russian). Russia, Moscow: Nauka. 1972, 368 pp.

[15] Harrison J.M. The diffusion approximation for the tandem service system in heavy traffic.UK. 1973. Adv.Appl.Probab., 10, 4, p. 887 - 905.

[16] Pavlov A.V. The random series of Fourier and its apply to the theory of prognosis-filtering.(In russian).Russia,Moscow : Moscow University nam.M.V.Lomonosov, mechanical-mathematical faculty,2000,ISBN 5-93839-002-8. 64 pp.

[17] Iglehart D.L., Whitt W. The multi- channel service systems in heavy traffic.I,II.UK. Adv.Appl.Probab. I,1970.v 2, 1, p. 150-177 ; II.1970, v 2. 2, p. 355-369.

[18] Pavlov A.V. Large deviation theorems for the chisquare test. Russia.Moscow :Adv. Math.Scien. 1996, V.51, 1(307), 159-160.

[19] Makarichev A.V. Two phase system with the identical service at different disciplines of service and high loading. Russia,Moscow:Jour. Izv.Ac.Sc. Tehnich.kibernet.1986, p. 115-118. 